\section{A randomised prospective double- masked exploratory study comparing combination photodynamic treatment and intravitreal ranibizumab vs intravitreal ranibizumab monotherapy in the treatment of neovascular age-related macular degeneration}

JH Vallance, B Johnson, MA Majid, S Banerjee, $K$ Mandal and CC Bailey

\begin{abstract}
Aims The aim of this study is to evaluate the effect of standard-fluence verteporfin photodynamic therapy (PDT) delivered on the first day of a ranibizumab regimen for choroidal neovascularisation secondary to age-related macular degeneration compared with ranibizumab monotherapy.

Methods Patients were randomised to sham or standard-fluence verteporfin PDT at baseline. The first of three monthly loading doses of ranibizumab was given on the same day, and thereafter patients received monthly treatment with ranibizumab as required. All patients underwent monthly visual acuity and OCT assessment and 3-monthly fluorescein angiography with follow-up to 1 year. Results In all, 18 patients were recruited. The PDT group gained a mean of 2.2 ETDRS letters at 1 year and the sham group gained a mean of 4.4 letters $(P=0.47)$. Both groups required a mean of 1.3 injections of ranibizumab following the 3-month loading phase. Fluorescein angiography at 1 month
\end{abstract}

demonstrated marked choroidal hypoperfusion in all patients treated with PDT with reduced choroidal perfusion persisting to month 12. This did not occur in the sham group.

Conclusion The addition of standard-fluence verteporfin PDT at baseline to a ranibizumab regimen conferred no benefit in terms of visual acuity or number of ranibizumab injections required at 1 year. The combination of these treatments resulted in persistent reduced choroidal perfusion, which raises potential safety concerns.

Eye (2010) 24, 1561-1567; doi:10.1038/eye.2010.84; published online 25 June 2010

Keywords: neovascularisation; macula; degeneration; treatment-medical; clinical trials

\section{Introduction}

Anti-vascular endothelial growth factor (anti-VEGF) monotherapy is now established as the standard of care for subfoveal choroidal
Clinical Research Unit, Bristol Eye Hospital, Bristol, UK

Correspondence: CC Bailey, Clinical Research Unit, Bristol Eye Hospital, Lower Maudlin Street, Bristol, Avon, United Kingdom. BS1 2LX, UK

Tel: + 01173424653 ;

Fax: +44 1173424891 .

E-mail: clare.bailey@ bristol.ac.uk

Received: 18 December 2009

Accepted in revised form: 30 April 2010

Published online: 25 June 2010 
neovascularisation ( $\mathrm{CNV}$ ) secondary to age-related macular degeneration (AMD). ${ }^{1}$ Although anti-VEGF therapies inhibit growth and permeability of new vessels and help prevent early CNV development, multiple retreatments are needed in most cases, and established CNV may be resistant to VEGF inhibition. ${ }^{2}$

Photodynamic therapy (PDT) with verteporfin has been shown to occlude established $\mathrm{CNV}$, but causes increased VEGF expression, which may lead to CNV recurrence. ${ }^{3}$ A combination of these two treatments could therefore potentially decrease the number of anti-VEGF treatments required to control $\mathrm{CNV}$.

The first study to investigate the combination of PDT and ranibizumab, the FOCUS study, ${ }^{4}$ raised safety concerns related to ocular inflammation, which was reduced following a protocol amendment changing the ranibizumab formulation. Subsequently, the PROTECT study ${ }^{5}$ has demonstrated that same-day administration of PDT and $0.5 \mathrm{mg}$ liquid ranibizumab is not associated with severe visual loss or ocular inflammation.

Uncontrolled trials ${ }^{3}$ and a large-scale analysis of registry data $^{6}$ have also suggested that combined therapy has the potential to reduce the number of anti-VEGF treatments required to control neovascular leakage.

\section{Materials and methods}

This single-centre, randomised pilot study compared intravitreal injection of ranibizumab and sham PDT treatment to a combination of intravitreal ranibizumab and standard-fluence verteporfin PDT delivered on the first visit. All patients received a further two monthly ranibizumab treatments, and were then retreated as required. The proportion of patients gaining at least 15 letters of ETDRS best-corrected visual acuity (BCVA) at 12 months compared with baseline was assessed as were the number of ranibizumab treatments at 6 and 12 months, BCVA after the initial loading phase of three monthly treatments, the proportion of patients gaining at least 10 letters at 12 months, the proportion of patients losing $<10$ and 15 letters at 12 months, and time to retreatment after the initial loading phase.

The study received local ethics committee and research and development approval. All patients gave written, informed consent before enrolment. (Clinical trial reference: ISRCTN42639823.)

\section{Patient selection and entry evaluations}

A screening visit comprised ocular examination, vision testing, medical history, OCT, and fluorescein angiography assessment. The full inclusion and exclusion criteria are shown in Table 1. The principal criteria were a visual acuity in the study eye of between 24 and 73 ETDRS letters, and an initial fluorescein angiogram demonstrating $\mathrm{CNV}$ secondary to AMD with evidence that the CNV extended under the geometric centre of the foveal avascular zone.

CNV were classified as either $100 \%$ classic/ predominantly classic or minimally classic/occult at baseline to allow stratification before randomisation. For patients with occult with no classic $\mathrm{CNV}$, clinical or angiographic evidence of recent disease progression was required for inclusion (Table 1). Patients who had previously received any other treatment for neovascular AMD were excluded.

\section{Visual assessment}

BCVA was measured in both eyes after refraction at baseline and at all follow-up visits using a standardised protocol by certified examiners using modified ETDRS charts. Contrast sensitivity and reading speed were assessed using Pelli-Robson and Bailey-Lovie methods at three monthly intervals.

\section{Imaging}

Fluorescein angiography was performed at baseline and repeated at months $1,3,6,9$, and 12 . The fluorescein angiographic features at baseline and follow-up were determined by two masked assessors (consultant ophthalmologists). OCT examinations were performed at every visit using the STRATUS OCT (Zeiss Meditech, Jena, Germany).

\section{Treatment protocol}

Patients were randomised $1: 1$ to receive initial standardfluence PDT or sham PDT. For the PDT group, the verteporfin infusion was covered by an opaque bag and given over $10 \mathrm{~min}$ at a dose of $6 \mathrm{mg} / \mathrm{m}^{2}$. PDT was given at a light dose of $50 \mathrm{~J} / \mathrm{m}^{2}$ and a fluence of $600 \mathrm{~mW} / \mathrm{m}^{2}$. For the sham group, the procedure was identical, but normal saline was substituted for verteporfin. Both the patient and the treating doctor were masked to the treatment given.

Intravitreal ranibizumab $(0.5 \mathrm{mg})$ was administered on the same day according to the Royal College of Ophthalmologists guidelines, ${ }^{7}$ and patients selfadministered a topical antibiotic (choramphenicol 0.5\%) four times a day for 3 days following treatment. Monthly assessment comprised visual acuity, OCT, and full ocular examination. All patients received further ranibizumab treatments at months 2 and 3 . Thereafter, ranibizumab was administered if there was a loss of more than 5 letters of BCVA associated with intraretinal or 
Table 1 Main inclusion/exclusion criteria

Inclusion criteria

Patients who at baseline:

- have a BCVA logMAR visual acuity in the study eye between 24 and 73 letters

- have a CNV of any type with the following characteristics as determined by fluorescein angiography:

1. Evidence that $\mathrm{CNV}$ extends under the geometric centre of the foveal avascular zone

2. CNV occupying at least $50 \%$ of the total lesion size

3. Total greatest linear dimension $\leqslant 5400 \mu$

4. No subfoveal atrophic change and no subfoveal fibrosis and a total area of fibrosis $\leqslant 50 \%$ of total lesion area

5. For occult with no classic $\mathrm{CNV}$, the lesion must demonstrate presumed recent disease progression as assessed by the investigator and defined at least one of the following criteria:

- Blood associated with the lesion at baseline

$-\geqslant 10 \%$ increase in GLD as assessed by fluorescein angiography in the past 3 months

- Loss of visual acuity in the last 3 months defined as either $\geqslant 5$ letters logMAR vision as determined by protocol refraction and protocol measurement or $\geqslant 2$ lines using a Snellen chart by standard examination

Exclusion criteria

Any previous CNV treatment in the study eye

Treatment with verteporfin in the non-study eye $<7$ days preceding enrolment

Any previous participation in a clinical trial involving anti-angiogenic drugs

Previous intravitreal drug delivery in the study eye

History of vitrectomy, glaucoma filtration surgery, corneal transplant or submacular surgery/other surgical intervention for AMD in the study eye, or any intraocular surgery in the study eye within 2 months of enrolment

Greater than mild non-proliferative diabetic retinopathy or any diabetic maculopathy

Previous retinal vascular occlusions

Subretinal haemorrhage that involves the centre of the fovea, if the size of the haemorrhage is either $>50 \%$ of the total lesion area or $>1$ disc area in size

$\mathrm{CNV}$ in either eye due to causes other than AMD

Retinal pigment epithelial tear involving the macula in the study eye

Active intraocular inflammation, or a history of uveitis

History of rhegmatogenous retinal detachment or macular hole (stage 3 or 4) in the study eye

Infectious conjunctivitis, keratitis, scleritis, or endophthalmitis in either eye

Aphakia or absence of the posterior capsule in the study eye, unless as a result of YAG posterior capsulotomy with previous posterior chamber intraocular lens implantation

Spherical equivalent of the refractive error in the study eye of more than $-8 \mathrm{D}$ of myopia or signs of pathologic myopia with a refraction of -4 to $-8 \mathrm{D}$. For subjects who have undergone cataract surgery in the study eye, a preoperative myopic refractive error of more than $-8 \mathrm{D}$

Uncontrolled glaucoma in the study eye, defined as intraocular pressure of $>30 \mathrm{~mm} \mathrm{Hg}$ despite anti-glaucoma medication Any concurrent intraocular condition in the study eye that, in the opinion of the investigator, is likely to require medical or surgical intervention during the study period to prevent or treat visual loss, or if allowed to progress untreated could contribute to a loss of at least two Snellen lines of BCVA over the study period

History of recent stroke or cardiac event, or uncontrolled angina or blood pressure

subretinal fluid on OCT, or a $>100 \mu \mathrm{m}$ increase in the mean central $1 \mathrm{~mm}$ retinal thickness, when compared to the measurement obtained following the three initial ranibizumab loading doses.

The incidence and severity of any ocular adverse events were documented. Treatment was withheld if a loss of BCVA of $>30$ letters, endophthalmitis, retinal detachment, retinal tear, or vitreous haemorrhage occurred within 14 days of treatment. Treatment was also withheld if intraocular surgery had been performed within 28 days, or there was any evidence of local or systemic infection. An intraocular pressure of $>45 \mathrm{~mm} \mathrm{Hg}$ at $1 \mathrm{~h}$ after injection was considered a severe adverse event.

\section{Statistics}

As this was a pilot study, there was insufficient evidence to precisely determine sample size in terms of visual acuity outcomes. The sample size was sufficient to give $80 \%$ power to detect a reduction in retreatments of two or more at 12-month follow-up. Statistical analysis was performed using SPSS for windows (http://www.spss.com, IBM headquarters, Chicago, IL, USA).

\section{Results}

Altogether, 18 patients met the study eligibility criteria and were randomised. All patients completed the study, 
Table 2 Baseline characteristics and results for verteporfin and sham PDT groups

\begin{tabular}{|c|c|c|}
\hline & Verteporfin group $(\mathrm{n}=9)$ & Sham PDT group $(\mathrm{n}=9)$ \\
\hline \multicolumn{3}{|l|}{ Baseline characteristics } \\
\hline Predominantly classic CNV (\%) & 44.4 & 44.4 \\
\hline Minimally classic/occult CNV (\%) & 55.6 & 55.6 \\
\hline Mean logMAR visual acuity & 50 letters & 55 letters \\
\hline Mean greatest linear dimension of lesion (microns) & 3185 & 2569 \\
\hline Mean central retinal thickness (microns) & 331 & 335 \\
\hline Mean reading speed (words per minute) & 126 & 172 \\
\hline Mean log contrast sensitivity score & 1.19 & 1.16 \\
\hline \multicolumn{3}{|l|}{ Results } \\
\hline Mean BCVA gain at 12 months & $\begin{array}{c}2.2 \text { (range }-8 \text { to }+24) \\
\text { letters }\end{array}$ & $\begin{array}{c}4.4 \text { (range }-11 \text { to }+20) \\
\text { letters }\end{array}$ \\
\hline Mean BCVA gain after initial treatment and loading phase & 3.1 letters & 6.5 letters \\
\hline Percentage of patients gaining $\geqslant 15$ letters at 12 months & 11.1 & 11.1 \\
\hline Percentage of patients gaining $\geqslant 10$ letters at 12 months & 11.1 & 33.3 \\
\hline Percentage of patients losing $<15$ letters at 12 months & 100 & 100 \\
\hline Percentage of patients losing $<10$ letters at 12 months & 100 & 88.9 \\
\hline Mean reduction in central foveal thickness on OCT at 12 months $(\mu \mathrm{m})$ & 138 & 103 \\
\hline Mean reading speed at 12 months (words per minute) & 136 & 171 \\
\hline Mean log contrast sensitivity score at 12 months & 1.26 & 1.21 \\
\hline Mean number of retreatments by 12 months & 1.3 (range $0-3$ ) & 1.3 (range $0-3$ ) \\
\hline Mean number of retreatments by 6 months & 0.2 & 0.4 \\
\hline Mean time to first retreatment (months) & 4.6 & 2.8 \\
\hline
\end{tabular}

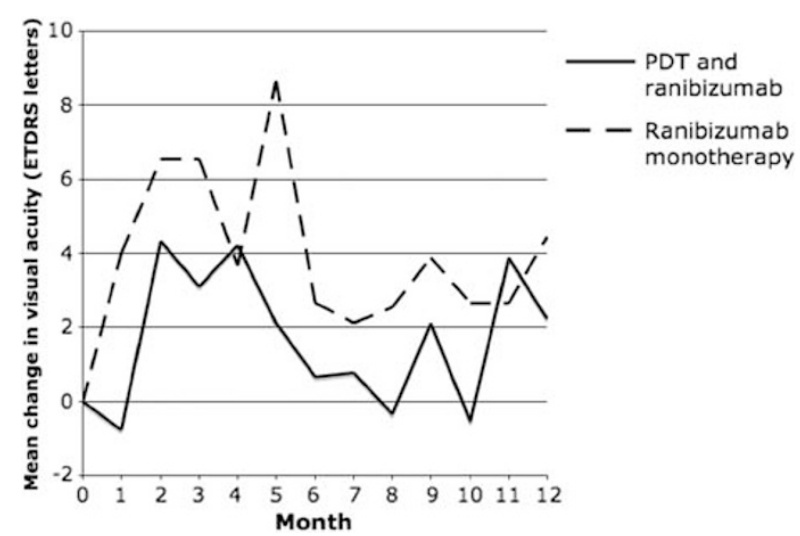

Figure 1 Mean change in ETDRS visual acuity for combination $\mathrm{PDT} /$ ranibizumab vs sham PDT/ranibizumab groups over the study period.

and no adverse events were recorded. Baseline characteristics and results are shown in Table 2.

\section{Visual acuity}

The PDT group gained a mean of 2.2 letters and the sham group 4.4 letters at 1 year (Table 2 ). This difference was not statistically significant $(P=0.47)$.

The PDT group gained a mean of 3.1 letters and the sham group 6.5 letters after the initial loading phase of the trial (month 4). After 1 month, five of the nine patients in the PDT group lost visual acuity, with three losing between 5 and 15 letters. In the sham group, two of nine patients

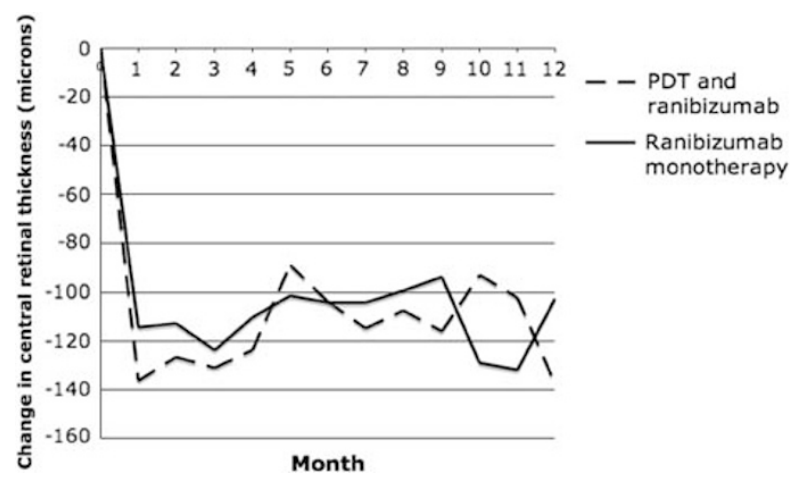

Figure 2 Mean change in central $1 \mathrm{~mm}$ retinal thickness for combination PDT/ranibizumab vs sham PDT/ranibizumab groups over the study period.

lost visual acuity 1 month after the first treatment, but no patients lost more than 5 letters. Mean visual acuity for the two groups over the year is shown in Figure 1.

\section{OCT and fluorescein angiography}

After 1 year, the mean central retinal thickness was reduced by $138 \mu$ in the PDT group and $103 \mu$ in the sham group $(P=0.57)$. A trend towards a greater reduction after first treatment in the PDT group was seen (Figure 2). All fluorescein angiograms in the PDT group and none of the sham group showed a circle of choroidal hypoflourescence corresponding to the area of PDT treatment at 1 month after initial treatment, and 


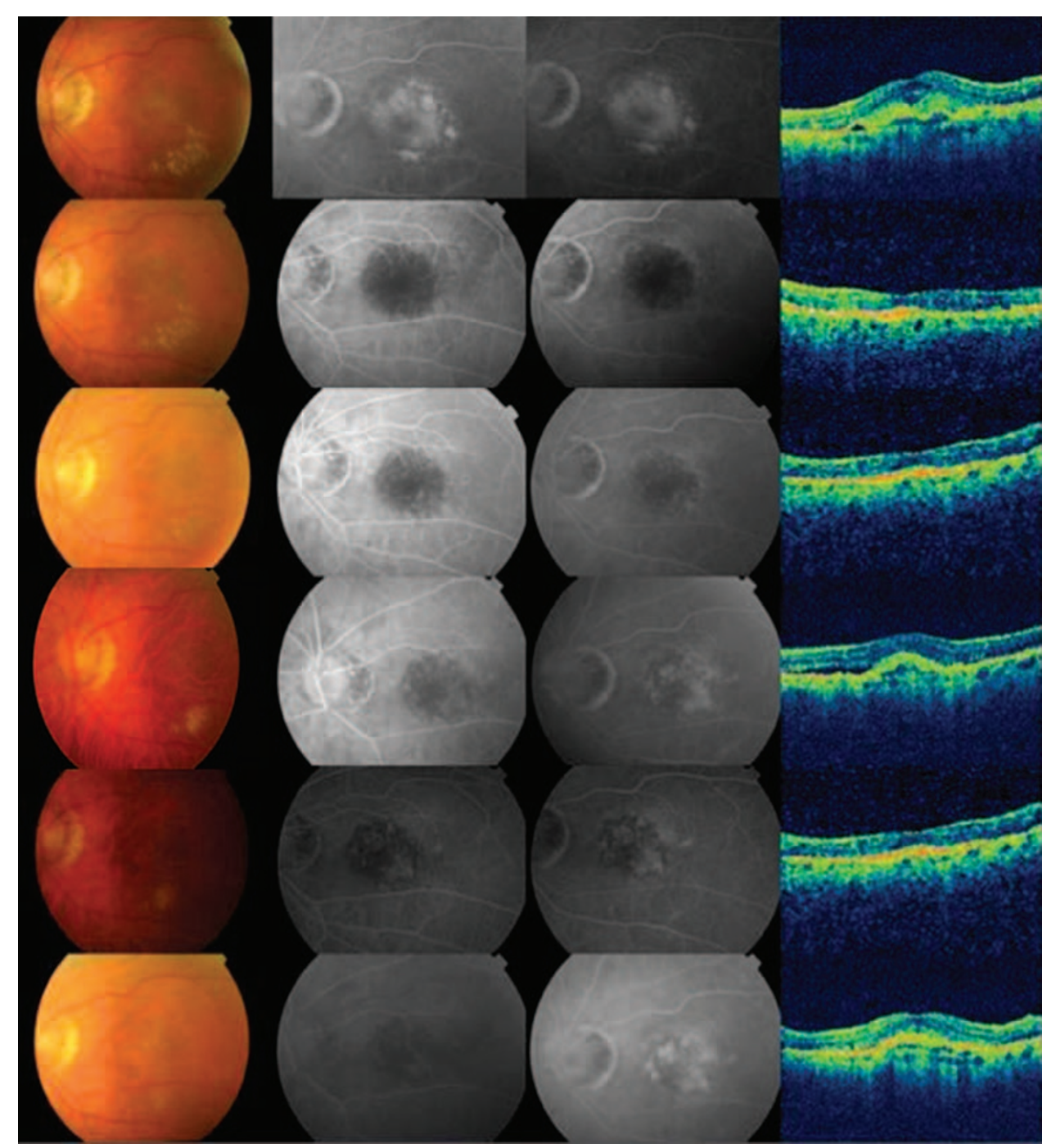

Figure 3 Serial fluorescein angiograms and OCT scans for one patient undergoing combination treatment. Images are from baseline and months 1, 3, 6, 9, and 12. Note the marked choroidal hypoperfusion at 1 month, with abnormal choroidal perfusion persisting over the 12-month follow-up. All combination-treated patients and none of the monotherapy group showed similar choroidal perfusion changes.

choroidal hypoperfusion persisted throughout the entire 12 months of the study (Figure 3).

\section{Treatment}

After the initial 3-month loading phase, both groups required a mean of 1.3 retreatments with ranibizumab over the 12 months of the trial. However, the mean time before the first retreatment was required was 4.6 months in the PDT group and 2.6 months in the sham group.

\section{Discussion}

Initial same-day PDT treatment conferred no benefit when compared with treatment with ranibizumab alone in terms of visual acuity, contrast sensitivity, or number of treatments required over the 1-year follow-up period. The initial increase in visual acuity during the first 3-month loading phase of ranibizumab treatment was blunted in the PDT group. The combination group had a lower mean reading score throughout the study and also had a very small (statistically insignificant) improvement in comparison to the monotherapy group. Reading speed is affected by different factors, including visual acuity and concentration levels.

In contrast to our findings, Pruente et $a l^{8}{ }^{8}$ using a similar study protocol but with a $0.3 \mathrm{mg}$ ranibizumab dosage, did demonstrate a reduced need for retreatment with ranibizumab in the group receiving PDT. However, this study also showed no difference between ranibizumab alone and combined therapy at 1 year in terms of visual acuity and a less rapid visual acuity gain 
in the combined treatment group. This trend towards reduction of the initial effect of ranibizumab when combined with PDT was also seen in the FOCUS study. FOCUS $^{4}$ is the largest trial of combined therapy to date, and mean visual acuity was lower in the group treated with PDT and ranibizumab than in the PDT-only group at 1 week and 1 month, but favoured the combinationtreated group after month 3 . However, unlike our study, FOCUS compared PDT only with combined PDT and ranibizumab.

In the combined treatment group of our study, striking choroidal hypoflourescence was observed on fluorescein angiography, and this persisted during the 12 months of follow-up, with recurrence of CNV occurring within this area of reduced choroidal perfusion. Reduced choroidal perfusion is a known consequence of PDT therapy as treatment temporarily closes small- and medium-sized choroidal vessels as well as $\mathrm{CNV} .{ }^{9}$ Concurrent treatment with both steroids ${ }^{10}$ and ranibizumab ${ }^{11}$ prolong reduced choroidal perfusion. The PROTECT study ${ }^{11}$

demonstrated reduced choroidal perfusion using indocyanine green angiography that persisted over 9 months of follow-up in 10 of 11 patients. The choroidal circulation did gradually reperfuse to some degree during this time, and CNV recurred only after reperfusion of the surrounding choriocapillaris. Microperimetry demonstrated improved retinal sensitivity despite reduced choroidal perfusion. No patients suffered severe visual loss in PROTECT and, in contrast to our findings, the mean visual acuity increased by 4.5 letters 1 month after initial treatment. It was concluded that the observed reduced choroidal perfusion did not appear to have any short-term functional significance. However, unlike our study, the PROTECT study was a small case series to evaluate the safety of same-day PDT and intravitreal ranibizumab and did not randomise patients to compare this regime with ranibizumab monotherapy. Furthermore, repeat PDT was administered if there was significant leakage found on fluorescein angiography at months 3, 6, and 9, and ranibizumab was only administered at baseline and at months 1,2 , and 3 .

It is not yet known whether inhibiting the process of vascular remodelling has any longer-term effects on the retinal pigment epithelium and retina. ${ }^{12}$ However, one study reporting 24-month data comparing PDT treatment with PDT and intravitreal triamcinolone ${ }^{11}$ demonstrated better visual acuity for the combination group in the first year, but progressive visual decline during the second year of follow-up. Reduced choroidal perfusion was still seen on angiography in the combined group at 24 months. Furthermore, the reduction of vision in the steroid-treated group was associated with atrophic chorioretinal and retinal pigment epithelium changes as demonstrated by retinal thinning on OCT and reduced macular fundus autofluorescence when compared with the PDT-only group. If ranibizumab is reducing choroidal reperfusion in a similar manner, it is at least possible that any potential benefit in terms of reduced treatment requirement could be at the cost of longer-term chorioretinal atrophy.

At present there is a lack of solid evidence that combination therapy is superior to ranibizumab monotherapy and, given concerns over potential adverse effects, longer-term results from larger randomised controlled trials are awaited. Strategies to limit reduced choroidal perfusion in combination therapy, such as reduced-fluence PDT with ranibizumab, are currently under investigation.

\section{Summary}

What was known before

- Ranibizumab monotherapy is the standard of care for neovascular age-related macular degeneration. However, monthly injections are required indefinitely for the best results. A combination of same-day administration of PDT and ranibizumab is not associated with severe visual loss or ocular inflammation. Non-randomised trials and retrospective analysis of registry data have suggested that a combination of PDT and ranibizumab may reduce the number of required ranibizumab injections.

What this study adds

- There is no additional benefit of combined standardfluence photodynamic therapy (PDT) and ranibizumab injections $v s$ ranibizumab alone for neovascular agerelated macular degeneration - There is persistent choroidal hypoperfusion at 1 year after combined PDT and lucentis injections, which raises potential safety concerns.

\section{Conflict of interest}

CC Bailey and MA Majid have received travel funding to attend ophthalmic conferences from Novartis UK.

\section{Acknowledgements}

We acknowledge Ms S Patra for her contribution as one of the assessors of fluorescein angiograms in this study and we also like to acknowledge the dedication of all the Clinical Research Unit staff at Bristol Eye Hospital. This study was funded by a block grant from Novartis UK.

\section{References}

1 Mitchell P, Korobelnik J-F, Lanzetta P, Holz FG, Pruente C, Schmidt-Erfurth UM et al. Ranibizumab (Lucentis) in neovascular age-related macular degeneration: evidence 
from clinical trials. Br J Ophthalmol 2010; 94(1): 2-13. Published Online First: 20 May 2009, doi:10.1136/ bjo.2009.159160.

2 Spaide RF. Rationale for combination therapy in age-related macular degeneration. Retina 2009; 29(6 Suppl): S5-S7.

3 Shah GV, Sang DN, Hughes MS. Verteporfin combination regimes in the treatment of neovascular age-related macular degeneration. Retina 2009; 29: 133-148.

4 Heier JS, Boyer DS, Ciulla TA, Ferrone PJ, Jumper JM, Gentile RC et al. Ranibizumab combined with verteporfin photodynamic therapy in neovascular age-related macular degeneration: year 1 results of the FOCUS Study. Arch Ophthalmol 2006; 124: 1532-1542.

5 Schmidt-Erfurth U, Wolf S, PROTECT Study Group. SameDay administration of verteporfin and ranibizumab $0.5 \mathrm{mg}$ in patients with choroidal neovascularisation due to agerelated macular degeneration. $\mathrm{Br} J$ Ophthalmol 2008; 92: 1628-1635.

6 Kaiser PK, Registry of Visudyne AMD writing committe. Verteporfin photodynamic therapy combined with intravitreal bevacizumab for neovascular age-related macular degeneration. Ophthalmology 2009; 116(4): 747-755.

7 Guidelines for Intravitreal Injections Procedure 2009. http://www.rcophth.ac.uk/about/publications/. (accessed on 21 May 2010).
8 Pruente C, Hatz K, Henrich PB, Braun B, Sacu S, Schneider U. A randomised double-masked study comparing lucentis monotherapy and pdt combined with lucentis therapy in patients with exudative amd: one year results for bcva and retreatment frequency. Presented at ARVO May 2009, abstract athttp://arvo.abstractsonline.com/plan/ SSResults.aspx. (accessed on 13 September 2009).

9 Schmidt-Erfurth UM, Michels S. Changes in confocal indocyanine green angiography through two years after photodynamic therapy with verteporfin. Ophthalmology 2003; 110: 1306-1314.

10 Piermarocchi S, Sartore M, Lo Giudice G, Maritan V, Midena E, Segato T. Combination of photodynamic therapy and intraocular triamcinolone for exudative age-related macular degeneration and long-term chorioretinal macular atrophy. Arch Ophthalmol 2008; 126(10): 1367-1374.

11 Kiss CG, Simader C, Michels S, Schmidt-Erfurth U. Combination of verteporfin photodynamic therapy and ranibizumab: effects on retinal anatomy, choroidal perfusion and visual function in the PROTECT study. $\mathrm{Br} J$ Ophthalmol 2008; 92: 1620-1627.

12 Schmidt-Erfurth U, Kiss C, Sacu S. The role of choroidal hypoperfusion associated with photodynamic therapy in neovascular age-related macular degeneration and the consequences for combination strategies. Prog Retin Eye Res 2009; 28: 145-154. 